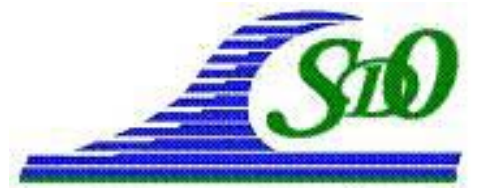

XI ${ }^{\text {èmes }}$ Journées Nationales Génie Côtier - Génie Civil

Les Sables d'Olonne, 22-25 juin 2010

DOI:10.5150/jngcgc.2010.104-P @ Editions Paralia CFL

disponible en ligne - http://www.paralia.fr - available online

\title{
Inversion du signal ADCP : application à la détermination des concentrations en matières en suspension dans l'estuaire de la Gironde
}

\author{
Jean-Paul PARISOT ${ }^{1}$, Lucile GLUARD ${ }^{\mathbf{2}}$, Guillaume DETANDT ${ }^{\mathbf{1}}$, \\ Eric MANEUX ${ }^{3}$, Julien MAS ${ }^{5}$, Georges OGGIAN ${ }^{1}$, Vincent SALBERT ${ }^{4}$, \\ Aldo SOTTOLICHIO ${ }^{1}$,
}

1. Université Bordeaux 1, EPOC UMR 5805, Av des facultés, 33405 Talence, France. parisot@epoc.u-bordeauxl.fr; a.sottolichio@epoc.u-bordeauxl.fr ; g.oggian@epoc.u-bordeauxl.fr

2. Université de Caen, UMR CNRS 6143, 24 rue des Tilleuls,14000 Caen, France. lucile.gluard@unicaen.fr

3. GEO TRANSFERT, Cellule de valorisation de l'UMR CNRS 5805 EPOC, Université Bordeaux 1, Avenue des Facultés, 33405 Talence cedex, France. e.maneux@epoc.u-bordeauxl.fr

4. Ginger, 11 rue Paul Baudry, 75008 Paris, France.

v.salbert@gingergroupe.com

5. Grand Port Maritime de Bordeaux,152 quai de Bacalan, 33082 Bordeaux, France. j-mas@bordeaux-port.fr

\section{Résumé :}

Depuis plus d'une dizaine d'années, les profileurs ADCP ne sont plus seulement utilisés comme courantomètres, mais leur intensité rétrodiffusée du signal est exploitée afin de quantifier la concentration de matières en suspension. Dans cette étude, nous appliquerons cette inversion du signal dans l'estuaire de la Gironde, connu pour ses concentrations en matières en suspension élevées et pour ses forts courants. Pour cela, il a été déployé un ADCP $600 \mathrm{kHz}$ lors de 3 campagnes de mesures. Des filtrations d'échantillons d'eau ont été réalisées, parallèlement, à des mesures optiques de turbidité (OBS). Ces jeux de données ont permis de calibrer l'ADCP en concentration massique. Le milieu étudié étant fortement concentré, une méthode de calcul itérative a été mise en place. Les résultats présentés dans cette étude étant tout à fait satisfaisants, il apparaît clairement que les ADCP ont le potentiel pour mesurer/estimer des profils de concentration de matières en suspension dans un environnement fortement concentré avec plus $1000 \mathrm{mg} \mathrm{l}^{-1}$ de MES.

Mots-clés :

ADCP - MES - Turbidité - Gironde 


\begin{abstract}
:
For more than a decade, ADCPs are not only used as current meters, but also the backscatter intensity of the signal is used to quantify the suspended matter concentration in natural waters. In the present work, we apply this signal inversion in the Gironde Estuary, which is very concentrated in suspended matter (over $1 \mathrm{~g} \mathrm{l}^{-1}$ ). A $600 \mathrm{kHz}$ ADCP from RDI was deployed during three field experiments. During the first campaign, filtered water samples were realised simultaneously to the ADCP records. During the second campaign, optical measurements of turbidity (OBS) were made. Those two data sets allowed us to calibrate our ADCP in mass concentration. This calculation allows us to create a suspended material concentration time series for each campaign. Those two series have been validated from the turbidity data sets (filtered samples and OBS). The results presented in this study are globally satisfactory with a minimum of calibration parameters, so they demonstrate the potential of ADCP to measure suspended sediment concentration in very concentrated environments.
\end{abstract}

\title{
1. Introduction
}

Les courantomètres acoustiques à effet Doppler (ADCP) sont de plus en plus utilisés pour quantifier les concentrations en matières en suspension dans les eaux naturelles. Le principe de mesure s'appuie sur l'inversion du signal acoustique dont l'intensité rétrodiffusée dépend des éléments présents dans le milieu (ici, les matières en suspension, biologiques et minérales). L'utilisation de ces méthodes est relativement mieux maîtrisée pour des sédiments non cohésifs, et dans des milieux faiblement concentrés. Notre étude a pour objectif de tester et d'exploiter l'intensité rétrodiffusée afin de quantifier la concentration en matières en suspension dans un estuaire fortement concentré $\left(>1 \mathrm{~g} \mathrm{l}^{-1}\right)$. Nous avons ainsi mis au point un algorithme de calcul de la concentration en matière en suspension, à partir des études de TESSIER (2006). Cette méthode a été testée et appliquée à plusieurs jeux de données disponibles dans l'estuaire de la Gironde (GLUARD, 2008 ; PARISOT et al., 2009). La méthode d'inversion est très sensible à la taille des particules, ce qui fait que la méthode pourrait potentiellement aider à déduire indirectement la taille moyenne des particules en suspension.

\section{L'instrument ADCP}

L'ADCP (Acoustic Doppler Current Profiler) est un courantomètre acoustique à effet Doppler. Il permet de mesurer et d'enregistrer des courants jusqu'à 2000 mètres de l'instrument, selon la fréquence de celui-ci. Des ondes sonores (pings) sont émises par l'ADCP ; elles sont diffusées sur les objets rencontrés sur leur passage (particules de sédiment ou zooplancton); ces ondes reviennent à l'émetteur qui devient récepteur, à une fréquence différente de celle d'émission, en raison de l'effet Doppler provoqué par les particules en mouvement. Dans notre étude, c'est l'intensité du signal rétrodiffusé qui sera utilisée, afin de quantifier les concentrations de matières en suspension (MES). 
Dans le cadre d'une utilisation normale de l'ADCP (i.e. courantomètre), ces canaux d'intensité ne sont pas analysés. Quatre transducteurs, indépendants, réalisent une mesure de l'intensité rétrodiffusée le long de chaque faisceau. Un signal est émis environ toutes les secondes. La discrétisation verticale dépend principalement de la taille des cellules (bins) définie par l'utilisateur. La mesure de la vitesse est calculée par auto corrélation du signal. Le "blank" Ro correspond à une zone non mesurée par l'ADCP, à cause de la vibration de la céramique après émission qui perturbe le champ de pression à proximité de l'ADCP. La mesure de $N R$ est faite a priori sur la dernière moitié d'une cellule.

\section{Principe et méthodes}

\subsection{L'équation du sonar}

La méthode d'inversion acoustique est basée sur l'équation du sonar dont la solution est $\mathrm{M}$, la quantité de matières en suspension exprimée à chaque niveau en $\mathrm{mg} \mathrm{l}^{-1}$ (TESSIER, 2006 ; TESSIER et al., 2008) :

$$
\begin{gathered}
10 \log _{10} M=N R-N E+40 \log _{10}(\psi R)-10 \log _{10}(\Psi L)+2 \int_{0}^{R}\left(\alpha_{w}+\alpha_{s}\right) d r-10 \log _{10}\left(\frac{\bar{\sigma}}{\rho_{s} \bar{v}_{s}}\right) \\
\alpha_{s}=\alpha_{v}+\alpha_{d}=8.686\left(\zeta_{v}+\zeta_{d}\right) M
\end{gathered}
$$

Dans cette équation, NR et NE sont respectivement les niveaux acoustiques reçus et émis, exprimés en $\mathrm{dB}$. Les 3 termes suivants expriment les pertes par transmission, dues à la divergence sphérique du faisceau, à l'absorption par l'eau et par la matière en suspension. Le dernier terme exprime la diffusion par les particules en suspension. Les 2 équations sont couplées par le terme d'absorption (par les sédiments) dépendant de la quantité $\mathrm{M}$ inconnue. Une méthode itérative très sensible à la taille moyenne des particules permet de résoudre ces 2 équations et d'obtenir le profil de MES. La méthode itérative permet, dans notre application avec 10 itérations d'obtenir une convergence à 99\%, car ce n'est pas le cas partout, dépendant de l'environnement étudié. L'itération est initiée avec un profil quelconque car la méthode converge rapidement. L'approximation qui est utilisée dans notre calcul, est une taille unique sur toute la colonne d'eau qu'il est difficile d'améliorer devant la complexité du milieu et la difficulté d'obtenir des profils. D'autre part, la taille équivalente est critique. Nous verrons que le résultat étant très sensible à ce paramètre, la méthode d'inversion ne fonctionne que dans une gamme très étroite de taille, ce qui nous permet (presque à l'aveugle) de déterminer une taille équivalente. 


\subsection{Développement d'un algorithme d'inversion}

Dans le cadre de cette étude nous avons utilisé un ADCP 600 kHz (modèle Workhorse $614 \mathrm{kHz}$ RDI). RDI donne pour leur ADCP $600 \mathrm{kHz}$, une fréquence $f=614,4 \mathrm{kHz}$, un niveau émis $N E=217,1 \mathrm{~dB}$ (que nous avons supposé constant), un diamètre de transducteur $a_{t}=101 \mathrm{~mm}$. Pour d'autres caractéristiques de l'appareil nous avons utilisé des valeurs moyennes issues des travaux de TESSIER (2006). Le bruit $\mathrm{Br}$, a été fixé à $85 \mathrm{~dB}$. Le niveau de bruit interne $N C o$ est le niveau de bruit (exprimé en counts) perçu par l'ADCP en réception seule dans un bassin d'eau. Comme il peut y avoir des bruits parasites, il est plus efficace de prendre la valeur minimale de l'intensité rétrodiffusée qui dans nos mesures varie de 35 à 80 counts. Il faut aussi fixer la distance du transducteur à la première cellule ou blank Ro. La taille des cellules, est définie par l'utilisateur. Pour le calcul des pertes par divergence sphérique, la distance de Fresnel utilisée est de $1.004 \mathrm{~m}$. Les caractéristiques du milieu sont issues de différentes sources bibliographiques sur la Gironde avec en moyenne : salinité $=7 \%, \mathrm{pH}=8$ et $\mathrm{T}=20^{\circ}$.

Les différents stades du calcul sont les suivants :

A. Un premier stade où l'on réalise la courbe de relation entre l'indice de rétrodiffusion volumique et la concentration de matières en suspension obtenue avec les jeux de données extérieurs (filtrations ou mesures optiques de turbidité). Cette étape permet de définir la concentration massique $M o$ de chaque cellule du profil d'ordre zéro. L'itération est initiée avec un profil quelconque car la méthode converge rapidement. Cette méthode est totalement inadaptée dans le milieu étudié car le signal qui augmente avec la MES décroit ensuite rapidement en raison de l'absorption et de la diffusion. La robustesse de la méthode itérative permet une grande latitude sur ce profil initial qui n'a pas beaucoup d'importance.

B. Dans un second stade, on calcule grâce à la concentration $M o$, le coefficient d'atténuation dû aux particules $\alpha_{s}$ dans chaque cellule. Ainsi on a un couple $\left(M, \alpha_{s}\right)$ pour chaque cellule.

C. Le troisième stade permet d'itérer sur la valeur de la concentration jusqu'à ce qu'elle converge. C'est-à-dire, que l'on prend la concentration $M o$ de la première cellule en partant de l'ADCP, on calcule ensuite l'atténuation due aux particules $\alpha_{s}$ dans cette cellule; puis on calcule la concentration de la deuxième cellule grâce au $\alpha_{s}$ précédent; on en déduit l'atténuation due aux particules de cette cellule ; puis on calcule la concentration de la troisième cellule, on en déduit l'atténuation due aux particules. Lorsque ce profil est arrivé à la dernière cellule, on le compare au précédent. Si entre deux itérations la valeur des MES varie de plus de $10^{-6}$ en valeur relative, on refait une itération, sinon on arrête, et on estime que le profil est cohérent. 


\section{Caractéristiques hydrosédimentaires du milieu étudié}

L'estuaire de la Gironde est un estuaire connu pour avoir des concentrations en matières en suspension très élevées $\left(>1 \mathrm{~g} \mathrm{l}^{-1}\right)$. Ses principales caractéristiques hydrosédimentaires sont tirées de CASTAING (1981). La combinaison de l'asymétrie de l'onde de marée et de la circulation résiduelle de densité favorise la formation d'un bouchon vaseux (BV). Sa position varie, en autres, en fonction de l'excursion de la marée ; c'est pour cela que le $\mathrm{BV}$ se décale vers l'amont au flot, et vers l'aval au jusant. La concentration en MES dans le $\mathrm{BV}$ peut dépasser $1 \mathrm{~g} \mathrm{l}^{-1}$ en surface et $10 \mathrm{~g} \mathrm{l}^{-1}$ au fond. Lorsque le courant faiblit, les MES décantent. Les connaissances sur les caractéristiques des particules sont plus rares en Gironde. Les caractéristiques de taille et nature des flocs sont moins documentées. Une étude de MANNING et al. (2000) a permis de montrer que sur 99 flocs, 63 étaient des microflocs de forme quasi ronde; il a été déterminé une masse volumique moyenne des différents types de flocs : $1160 \mathrm{~kg} \mathrm{~m}^{-3}$ pour les microflocs et $1027 \mathrm{~kg} \mathrm{~m}^{-3}$ pour les macroflocs ainsi que la taille associée (tableau 1); et que 56,6\% de la concentration en MES est contenue dans les macroflocs. Ces données nous ont permis de déterminer la taille et la densité des particules présentes dans l'environnement étudié. La densité la plus appropriée correspond à des microflocs. Quant à une taille, elle a été ajustée à des valeurs variant de 10 à $35 \mu \mathrm{m}$ suivant le site de mesures.

Tableau 1. Tableau récapitulatif des résultats de MANNING et al. (2000).

\begin{tabular}{llll}
\hline & macroflocs & microflocs & prticule seule \\
\hline Masse volumique $\left(\mathrm{kg} \mathrm{m}^{-3}\right)$ & 1027 & 1160 & 2600 \\
Diamètre $(\boldsymbol{m})$ & $3,75 \times 10^{-4}$ & $2,5 \times 10^{-5}$ & $5 \times 10^{-6}$ \\
\hline
\end{tabular}

\section{Campagnes de mesures}

Pour cette étude, nous avons à notre disposition trois jeux de données provenant de campagnes différentes. Elles ont été collectées respectivement aux points PK 30 (SOMLIT -Service d'Observation en Milieu LITtoral-), PK 48 (REAGIR2 -REActions Chimiques dans la GIRonde), et en divers endroits entre les points notés A1 et B3 (GPMB -Grand Port Maritime de Bordeaux-) sur la figure 1.

- Le premier jeu de données a été acquis lors de la campagne SOMLIT le 30 janvier 2008, période de mortes eaux, au point PK 30 (figures 1 et 2). L'ADCP a été mouillé pendant $8 \mathrm{~h} 30$, réalisant des mesures toutes les secondes. La taille des cellules choisie était de $50 \mathrm{~cm}$, et le blank Ro était de $1.07 \mathrm{~m}$. Parallèlement, des prélèvements d'eau avec une bouteille Niskin ont été effectués, à 1,3 et $8 \mathrm{~m}$ de profondeur. Un profil vertical de prélèvements d'eau a été effectué tous les mètres. Grâce à ces échantillons, des filtrations ont servi à calibrer l'ADCP. 


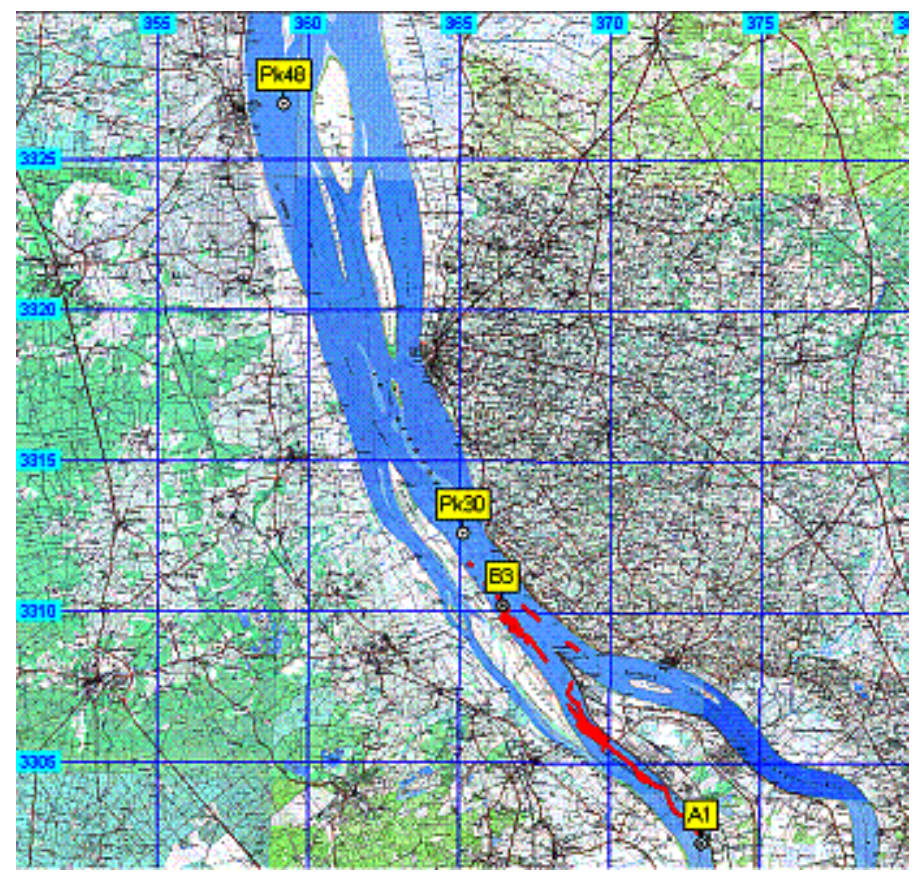

Figure1. Situation des sites de mesures ADCP utilisées dans ce travail.

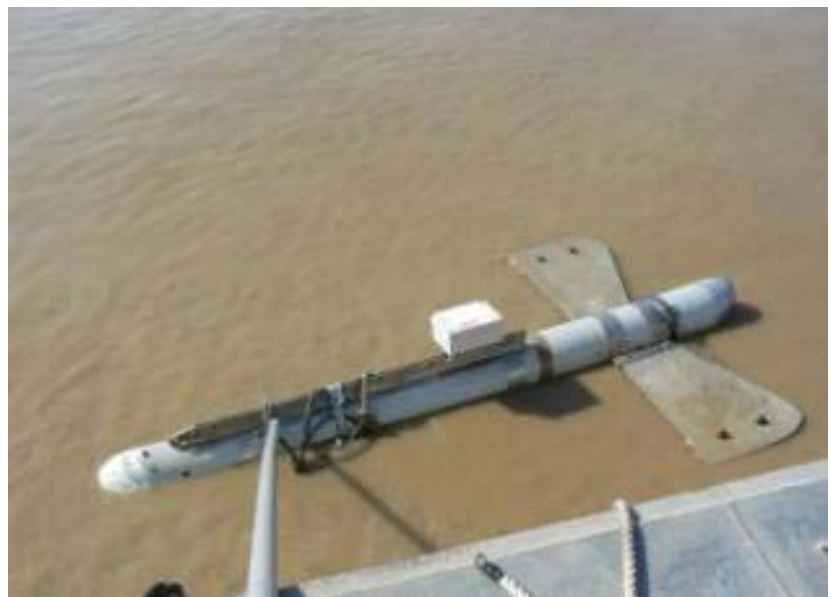

Figure 2. Installation de l'ADCP en-dessous d'un "requin" flottant.

- Le second jeu de données est issu de la campagne REAGIR2, qui s'est déroulée les 5, 6 et 7 octobre 2004, période de mortes eaux, au point PK 48 (figure 1). L'ADCP a été mouillé de 16h18 le 05/10/04 à 12h54 le 7/10/04, réalisant donc des mesures sur une période de $44 \mathrm{~h} 36$, toutes les secondes environ. Parallèlement, des profils de mesures de turbidité ont été effectués toutes les heures grâce à un OBS (Optical Backscattering Sensor) de 22h24 le 05/10/04 à 10h58 le 06/10/04. L'OBS a donné des profils (après traitement) de concentration environ toutes les heures. 


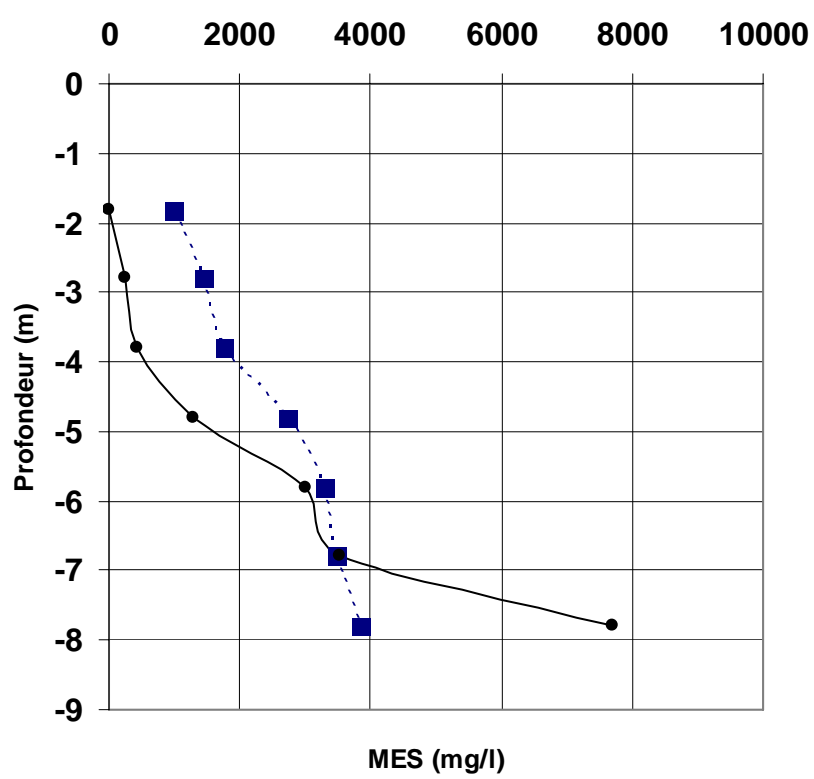

Figure 3. Comparaison entre le profil ADCP (traits pleins) et le profil OBS (tirets).

- La figure 3 montre la similitude d'estimations réalisées entre les 2 méthodes : l'allure des profils est respectée, l'ordre de grandeur des concentrations aussi; la calibration ADCP semble donc être un bon outil quant à la quantification de la concentration de matières en suspension jusqu'à des valeurs de $8 \mathrm{~g} \mathrm{l}^{-1}$, qui sont les valeurs maximales que nous avons rencontrées.

- Le troisième jeu (GPMB) a été obtenu en 2009 dans le cadre du suivi d'un dragage expérimental par la technique JETSED réalisée par Ginger Environnement avec la participation de la cellule de transfert GEO Transfert et le laboratoire EPOC pour le compte du Grand Port Maritime de Bordeaux. L'ADCP, a été mis en œuvre à l'aide d'un flotteur trainé par une vedette (figure 1). Des profils OBS et des prélèvements d'eau complétaient les mesures ADCP.

\section{Résultat obtenus pendant la campagne GPMB}

\subsection{Calibrations}

Les 2 premiers jeux de données ont permis de tester la méthode d'inversion qui a été appliquée complètement à la $3^{\text {ème }}$ série que nous développons ici (PARISOT et al., 2009). Le nombre et le type d'enregistrements réalisés au cours des trois jours sont détaillés dans les tableaux 2 et 3 avec en particulier les mesures réalisées le 17/09/2009. Pour mesurer les profils verticaux de turbidité, un OBS a été utilisé en parallèle avec une sonde multi-paramètres. Trois campagnes ADCP ont été réalisées les 17, 21 et 22 septembre 2009. La carte de la figure 1 présente les trajectoires du bateau ainsi que les points fixes de mesure qui sont répartis entre les points A1 et B3. En plus, $40 \mathrm{~kg}$ de 
crème de vase fraîche ont été récoltés dans la zone intertidale. La calibration du signal OBS (en volt) avec des mesures de concentration en MES de solutions expérimentales, réalisées par filtration, montre une saturation du capteur autour de $7 \mathrm{~g} \mathrm{l}^{-1}$ et une bonne corrélation qui permet de valider les mesures.

Tableau 2. Synthèse des mesures effectuées en septembre 2009.

\begin{tabular}{lllllll}
\hline Date & $\begin{array}{l}\text { Nombre de } \\
\text { sites }\end{array}$ & $\begin{array}{l}\text { Profils } \\
\text { OBS }\end{array}$ & Points fixes & $\begin{array}{l}\text { Nombre de } \\
\text { trajets }\end{array}$ & $\begin{array}{l}\text { Nombre de } \\
\text { profils } \\
\text { ADCP }\end{array}$ & $\begin{array}{l}\text { Nombre de } \\
\text { transects } \\
\text { ADCP }\end{array}$ \\
\hline $\mathbf{1 7 / 0 9 / 2 0 0 9}$ & 8 & 8 & 8 & 1 & 8 & 1 \\
$\mathbf{2 1 / 0 9 / 2 0 0 9}$ & 13 & 13 & 13 & 2 & 12 & 2 \\
$\mathbf{2 2 / 0 9 / 2 0 0 9}$ & 12 & 12 & 12 & 1 & 9 & 3 \\
\hline
\end{tabular}

Tableau 3. Synthèse des mesures effectuées le 17 septembre 2009.

\begin{tabular}{|c|c|c|c|c|c|c|c|}
\hline Site & Heure & $\begin{array}{c}\text { Profondeur } \\
\text { sonde }(m)\end{array}$ & $\begin{array}{c}\text { Nombre de } \\
\text { mesures } \\
\text { OBS } \\
\end{array}$ & $\begin{array}{c}\text { MES } \\
\text { minimale } \\
\left(\mathrm{g} l^{-1}\right) \\
\end{array}$ & $\begin{array}{c}\text { Profondeur } \\
\text { de saturation } \\
(\mathrm{m})\end{array}$ & $\begin{array}{l}\text { Durée } \\
\text { ADC } \\
(\min ) \\
\end{array}$ & $\begin{array}{c}\text { Nombre } \\
\text { de profils } \\
\text { ADCP }\end{array}$ \\
\hline$A 1$ & $13: 17$ & 6.2 & 45 & 1.9 & 4.0 & 12.2 & 377 \\
\hline$A 2$ & $12: 42$ & 7.5 & 56 & 1.8 & 6.0 & 10.5 & 318 \\
\hline$A 3$ & $12: 12$ & 6.5 & 75 & 1.6 & 3.5 & 12.1 & 336 \\
\hline$A 4$ & $11: 37$ & 6.0 & 53 & 1.0 & - & 21.2 & 348 \\
\hline Transect & $13: 26$ & - & - & - & - & 20.5 & 786 \\
\hline
\end{tabular}

6.2 Sensibilité à la taille des particules.

La méthode est très sensible à la taille des particules car la diffusion varie comme la puissance 16 du rayon des macros flocs. Dans le script développé on utilise comme paramètre d'ajustement la taille moyenne. Une taille moyenne trop petite donne des quantités de MES trop surestimées et inversement une taille trop grande conduit à des concentrations en MES trop faibles. Les figures $4 a, 4 b$ et $4 c$ (correspondant à l'inversion du profil relevé le 17 décembre au point A1) présentent successivement la quantité de MES nécessaire pour reproduire le signal ADCP avec, respectivement, des rayons de 9,12 et $15 \mu \mathrm{m}$. On constate que la valeur de $12 \mu \mathrm{m}$ est un bon compromis. Les profils présentés sur les figures $4 \mathrm{a}, 4 \mathrm{~b}$, et $4 \mathrm{c}$, correspondent à quelques minutes de mesures réalisées dans le panache créé en temps réel par le rejet de la drague. Il s'agit d'un panache instantané et qui n'a pas l'allure d'un profil de matière en suspension standard puisque l'ADCP est très proche du rejet. De plus, il se déplace et enregistre des données correspondant à des positions différentes. 


\section{XI $I^{\text {èmes }}$ Journées Nationales Génie Côtier - Génie Civil \\ Les Sables d'Olonne, 22-25 juin 2010}

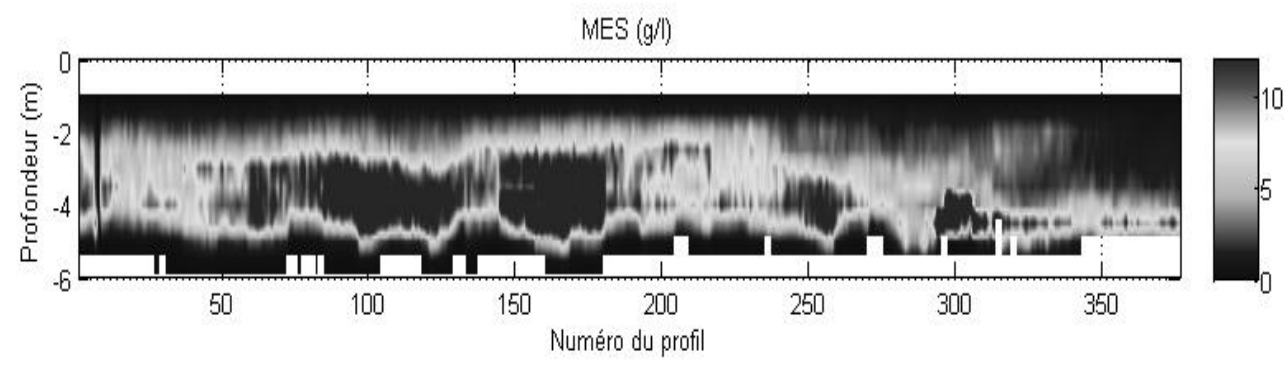

Figure 4a. Inversion du profil Al avec des tailles de particules de $9 \mu \mathrm{m}$.

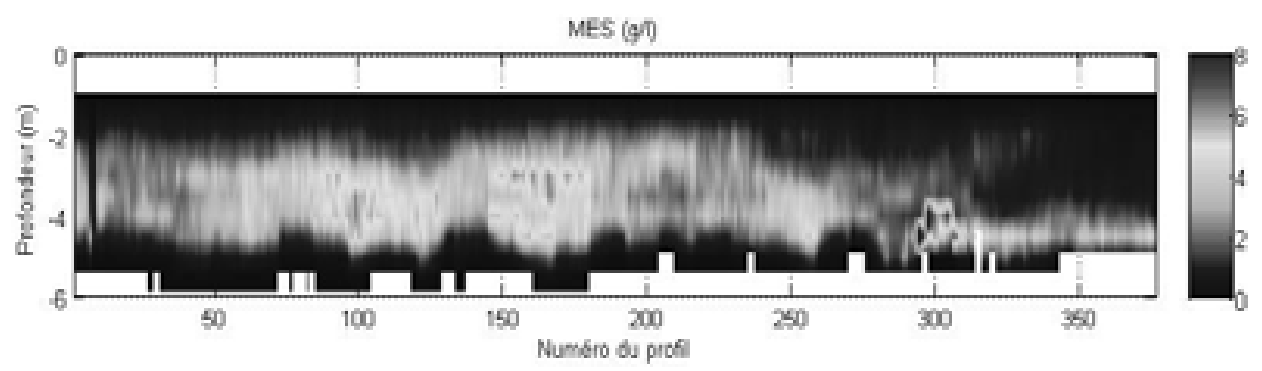

Figure 4b. Inversion du profil Al avec des tailles de particules de $12 \mu \mathrm{m}$.

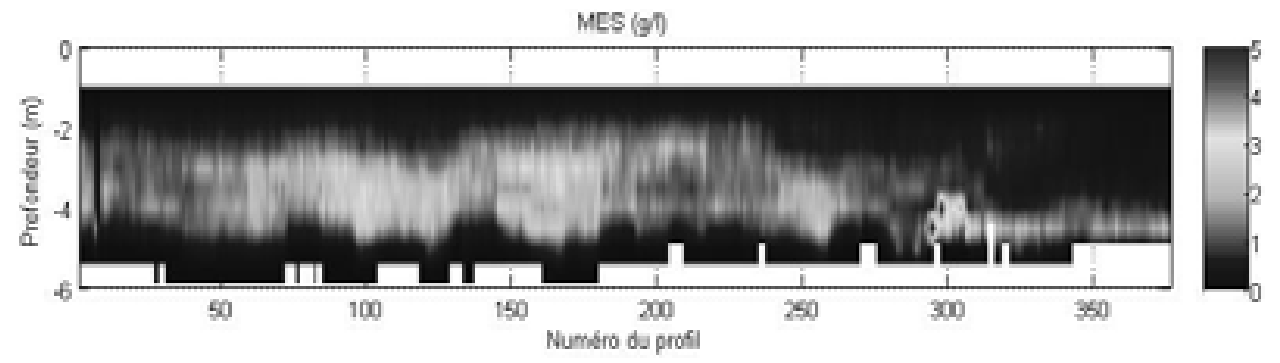

Figure 4c. Inversion du profil Al avec des tailles de particules de $15 \mu \mathrm{m}$.

\section{Discussion}

La méthode itérative permet d'obtenir la concentration massique sur toute la colonne d'eau. Ces profils ont été validés avec ceux obtenus par filtration ou mesures optique de turbidité. Ayant été jugés satisfaisants, l'algorithme a été appliqué sur tous les profils ADCP permettant ainsi d'obtenir une variation temporelle de la concentration en matière en suspension. Après comparaison avec les jeux de données de mesure de turbidité extérieurs, l'inversion du signal rétrodiffusé par un ADCP semble être un outil judicieux pour la détermination de concentration en matières en suspension, dans un environnement fortement concentré et soumis à de forts courants, moyennant une bonne connaissance des caractéristiques des particules. La méthode d'inversion est très sensible à la taille des particules. Aussi, si celle-ci n'est pas connue a priori, son ajustement lors de la calibration du signal pourrait potentiellement aider à déduire les tailles équivalentes moyennes des particules en suspension dans le milieu. 
Thème 3 - Instrumentation, mesures, imagerie et télédétection

\section{Références bibliographiques}

CASTAING P. (1981). Le transfert à l'océan de suspensions estuariennes - Cas de la Gironde. Thèse, Université de Bordeaux 1, $530 \mathrm{p}$

GLUARD L. (2008). Inversion du signal ADCP : quantification des matières en suspension dans la Gironde. Master 2 ENVOLH. Université de Bordeaux1.

MANNING A.J., DYER K.R., CHRISTIE M.C. (2000). Properties of macroflocs in the seaward limit of the Gironde Estuary. Proc. VII Colloque International d'Océanoraphie du Golfe de Gascogne, Biarritz, France, 4-6 April 2000.

PARISOT J.P., GLUARD L., SOTTOLICHIO A., OGGIAN G. (2009). Estimation of suspended solids concentrations from backscatter measured by acoustic Doppler current profiler in a highly turbid estuary. $10^{\text {th }}$ INTERCOH Conference, May 3-8 2009, Rio de Janeiro/Paraty-Brésil.

TESSIER C. (2006). Caractérisation et dynamique des turbidités en zone côtière : l'exemple de la région marine Bretagne sud. Thèse de doctorat, Université de Bordeaux 1, $428 \mathrm{p}$.

TESSIER C., LE HIR H., LURTON L. (2008). Estimation de la matière en suspension à partir de l'intensité rétrodiffusée des courantomètres acoustiques à effet Doppler, C.R.A.S. Geosciences, vol 340(1), pp. 57-67. doi:10.1016/j.crte.2007.10.009 